\title{
Cirurgia da aorta descendente e tóraco-abdominal com técnica de oclusão proximal isolada da aorta (“open distal")
}

Bayard GONTIJO FILHO* ${ }^{*}$ Fernando Antônio FANTINI*, Juscelino Teixeira BARBOSA*, Joăo Alfredo PAULA E SILVA*, Carla de OLIVEIRA*, Leonardo F. DRUMOND*, Eduardo P. ALCOCER*, Mário O. VRANDECIC*

RBCCV $44205-267$

GONTIJO FILHO, B.; FANTINI, F. A.; BARBOSA, J. T.; PAULA E SILVA, J. A.; OLIVEIRA, C.; DRUMOND, L. F.; ALCOCER, E. P.; VRANDECIC, M. O. - Cirurgia da aorta descendente e tóraco-abdominal com técnica de oclusăo proximal isolada da aorta ("open distal"). Rev. Bras. Cir. Cardiovasc., 10 (3): 119 125,1995

RESUMO: O presente relato apresenta a experiéncia obtida em um período de 4 anos (jan/91 - jan/95) com a técnica "open distal" descrita por Cooley, em $1990^{3}$, para tratamento cirúrgico de doenças da aorta torácica descendente e aorta tóraco-abdominal. Nesse periodo foram realizadas 35 operaçōes (25 para aorta descendente e 10 para aorta tóraco-abdominal). Os aneurismas de origem degenerativa $\theta$ as dissecçóes aórticas ocorreram em frequência semelhante $(48,5 \%$ e $40 \%$, respectivamente); em 8 pacientes havia rotura da aorta, parcialmente tamponada pelo pulmão ( 5 casos), órgăos abdominais ( 2 casos) e esófago ( 1 caso). O acesso círúrgico foi obtido por toracotomia póstero-lateral esquerda ou tóraco-freno-laparotomia. Heparina foi usada na dose de $1.5 \mathrm{mg} / \mathrm{kg}$ e todo sangue do campo cirúrgico fol coletado a um reservatório e reinfundido pela veia femoral. Houve $4(11.4 \%)$ óbitos hospitalares e $2(5,8 \%)$ pacientes portadores de aneurisma tóracoabdominal desenvolveram paraplegia. A morbi-mortalidade do grupo foi diretamente relacionada à condiçâo clínica per-operatória e à extensão do segmento ab́rtico acometido. Na nossa opiniăo, a técnica "open distal" é um método alternativo simples e eficaz no tratamento cirúrgico das doenças da aorta descendente e tóracoabdominal. Em casos de ressecçōes extensas com periodos longos de oclusão aórtica, métodos para proteçāo medular deverão ser avaliados.

DESCRITORES: Aorta descendente, cirurgia. Aorta tóraco-abdominal, cirurgia. Cooley, técnica de, "open distal", "Open distal", técnica de Cooley.

\section{INTRODUÇĀO:}

O tratamento cirúrgico das doenças da aorta torácica descendente e tóraco-abdominal apresenta um indice de morbi-mortalidade ainda elevado. quando comparado a outras intervençōes realizadas na aorta ascendente e aorta abdominal infrarenal. Os danos neurológicos originados pela isquemia medular e a insuficiência renal săo complicaçōes graves que nāo conseguiram ser eliminadas, a despeito do acúmulo de experiência e das novas técnicas propostas. Por este motivo, não existe consenso na literatura para definição do melhor procedimento cirúrgico nesses pacientes.
De modo genérico, podemos dizer que a proposta de manutenção da perfusāo distal da aorta, seja por circulaçāo extracorpórea ou por shunt passivo (tipo Gott), é contraposta por outros grupos que preconizam a oclusão simples da aorta, sem métodos adicionais para proteçāo medular e dos órgãos abdominais.

De 1990 , COOLEY ${ }^{3}$ descreveu uma alternativa técnica de substituiçāo da aorta torácica apenas com oclusão proximal da aorta ("open distal") com exsangûinação pelo coto aórtico distal. Os promissores resultados iniciais obtidos por seu grupo nos levaram a iniciar uma experiência com esta técnica

Trabalho realizado no Departamento de Cirurgia do Biocor Hospital de Doenças Cardiovasculares. Belo Horizonte, MG, Brasil. Apresentado ao $22^{\circ}$ Congresso Nacional de Cirurgia Cardiaca. Brasilia, DF, 30 de março a $1^{\circ}$ de abril, 1995.

* Do Departamento de Cirurgia do Biocor Hospital de Doenças Cardiovasculares.

Endereço para correspondéncia: Bayard Gontijo Filho. Caixa Postal 106. CEP 30161-970 Belo Horizonte, MG, Brasil. 
GONTIJO FILHO, B.; FANTINI, F. A.; BARBOSA, J. T.; PAULA E SILVA, J. A.; OLIVEIRA, C.; DRUMOND, L. F.; ALCOCER, E. P.; VRANDECIC, M. O. - Cirurgia da aorta descendente e tóraco-abdominal com técnica de oclusão proximal isolada da aorta ("open distal"). Rev. Bras, Cir. Cardiovasc., $10(3): 119-125,1995$.

a partir de 1991. O presente relato tem por objetivo apresentar nossa experiência ao longo desses 4 anos.

\section{CASUISTICA E MÉTODOS}

\section{A) Dados Clinicos}

No período de jan/91 a jan/95, 34 pacientes portadores de doenças da aorta descendente $e$ tóraco-abdominal foram submetidos a correção cirúrgica com a técnica "open distal". Um paciente foi submetido a 2 intervençōes, sendo a técnica empregada 35 vezes, com 25 substituiçōes da aorta descendente e 10 da aorta tóraco-abdominal. Este grupo năo foi consecutivo, uma vez que, nesse período, 5 pacientes foram operados com hipotermia profunda e parada circulatória para correçāo de lesōes mais complexas desse mesmo segmento ab́rtico. A idade dos pacientes variou de 25-76 anos, com média de 50,6 , havendo predominância do sexo masculino $(70,5 \%)$. A doença prevalente no grupo que motivou o tratamento cirúrgico foi o aneurisma de origem degenerativa $(48,5 \%)$, seguido pelas dissecçōes aórticas $(40,0 \%)$ (Tabela 1). Em 2 pacientes, a cirurgia foi indicada para substituição de uma prótese antiga, sendo em 1 por infecção e em outro devido a embolizaçōes repetidas nos membros inferiores por desgarramento da "pseudo-íntima" de prótese implantada 10 anos antes, inclusive com oclusão total da bifurcação da aorta abdominal em uma ocasiāo.

Quanto à condiçāo clínica pré-operatória, 8 $(22,8 \%)$ pacientes apresentavam rotura da aorta parcialmente tamponada pelo pulmăo (5 casos), órgãos abdominais (2 casos) e esôfago (1 caso); 2 desses pacientes foram operados em vigência de importantes alteraçōes hemodinâmicas. Apesar de 6 pacientes demonstrarem alterações laboratoriais pré-operatórias da funçảo renal, apenas 2 foram operados em franca insuficiência renal anúrica. Sete pacientes eram sabidamente portadores de lesōes das coronárias, sendo que 4 deles já haviam sido submetidos previamente a revascularizaçāo do miocárdio. Um paciente portador de sindrome de Marfan, que havia sido submetido a correçāo de dissecçăo aguda da aorta ascendnete 2 anos antes, teve expansăo rápida com rotura da aorta torácica, sendo feita substituiçăo da aorta descendente; no $8^{\circ}$ dia de pós-operatório apresentou dor de forte intensidade, acompanhada de hipotensăo e evidência radiológica de rotura aórtica ao nivel da anastomose distal. Foi, então, reoperado com substituição do restante da aorta tóraco-abdominal (Figura 1).
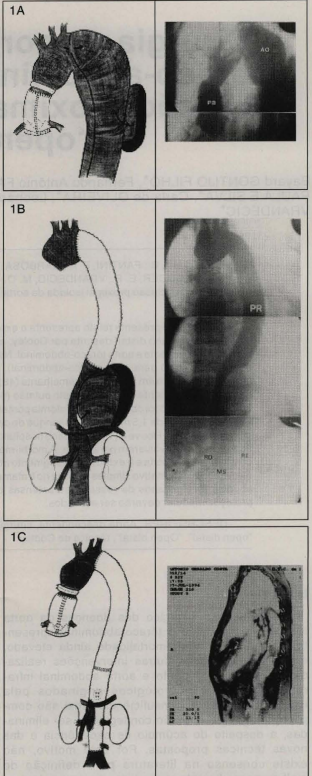

Fig. 1 - A: Aortografia em paciente portador de sindrome de Marian submetido à correção de dissecçâo aguda da aorta (2 anos) e com rotura na aorta descendente. B: Controle angiográfico no periodo de pós-operatório imediato $\left(8^{\circ} \mathrm{DPO}\right)$ com evidências de persisténcia da dissecçło aórtica e rotura ao nivel da anastomose distal. C: Aspecto final da correçảo com detathes da ressonância nuclear magnética pós-operatória. 
GONTIJO FILHO, B.; FANTINI, F. A.;BARBOSA, J. T.; PAULA E SILVA, J. A.; OLIVEIRA, C.; DRUMOND, L. F.; ALCOCER, E. P; VRANDECIC, M. O. - Cirurgia da aorta descendente e tóraco-abdominal com técnica de oclusão proximal isolada da aorta ("open distal"). Rev, Bras. Cir. Cardiovasc., 10 (3): 119-125, 1995.

TABELA 1

DISSECCÄO DE ANEURISMAS - AORTA DESCENDENTE E TÓRACO-ABDOMINAL PELA TÉCNICA "OPEN-DISTAL" $N=35$ - DADOS CLINICOS

\begin{tabular}{|c|c|c|c|}
\hline $\begin{array}{l}\text { DADOS DOS PACIENTES } \\
(\mathrm{m}=34)\end{array}$ & $\begin{array}{l}\text { Idade: } 25 \cdot 76 \text { anos } \\
\text { Sexo: }{ }^{\text {[masc. } 24} \\
\text { [fem. } 10\end{array}$ & $\begin{array}{l}(m=50,6) \\
(70,5 \%) \\
(29,5 \%)\end{array}$ & \\
\hline -TOPOGRAFIA & $\begin{array}{l}\text { Aorta Descendente } \\
\text { Aorta Tóraco-Abdominal }\end{array}$ & & $\begin{array}{l}=25 \\
=10\end{array}$ \\
\hline -ETIOLOGIA & $\begin{array}{l}\text { - Aneurisma Degenerati } \\
\text { Dissecção de Aorta } \\
\text { : Aneurisma Traumático } \\
\text { - Substituição de Prótes }\end{array}$ & $\begin{array}{l}\text { ativo } \\
\text { aguda } \\
\text { crônica } \\
\text { co } \\
\text { ese }\end{array}$ & $\begin{array}{l}=17 \\
=6 \\
=8 \\
=2 \\
=2\end{array}$ \\
\hline
\end{tabular}

\section{B) Técnica Cirúrgica}

Para anestesia endotraqueal foi empregado tubo de duplo lume para se obter colapso do pulmão esquerdo diminuindo, assim, o traumatismo sobre o parênquima pulmonar. $O$ acesso cirúrgico foi por toracotomia póstero-lateral ou tóraco-frenolaparotomia, dependendo da extensăo da lesăo. Nas doenças acometendo todo o trajeto da aorta descendente, foram realizadas duas incisőes intercostais $\left(4^{2} \mathrm{e} 7^{2}\right.$ espaço intercostal) pela mesma incisão

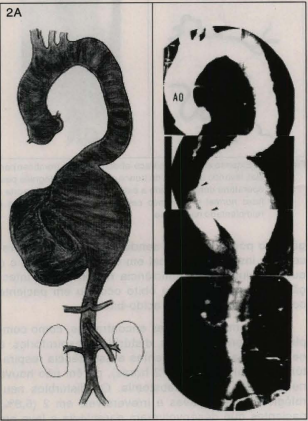

cutânea. Nas tóraco-freno-laparotomias o diafragma foi incisado radialmente com exposição da aorta abdominal por via retroperitoneal.

Heparina na dose de $1,5 \mathrm{mg} / \mathrm{kg}$. Aplicada pinça proximal e incisão da aorta promovendo a exsangüinaçăo do paciente com controle imediato de pressão arterial sem risco de sobrecarga ventricular aguda. Retirada de todo material trombótico; aspirado o sangue do campo cirúrgico para um reservatório de cardiotomia e reinfundido por uma cânula colocada na veia femoral esquerda. $\mathrm{Na}$ maior parte dos casos foi empregada prótese de Dacron impregnada de colágeno. Em paciente portadora de aneurisma sacular a correção foi com retalho de pericárdio bovino. A liberação da pinça da aorta era realizada lentamente, após infusāo de bicarbonato de sódio. Durante a correçāo cirúrgica raramente foram utilizadas drogas vasoativas, sendo o controle hemodinâmico mediado pelo circuito de reinfusão sangüínea. Nas substituiçōes da aorta

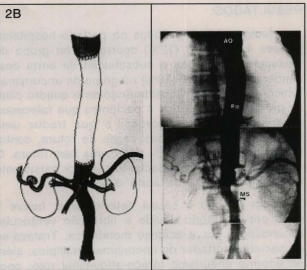

Fig. 2. A: Grande aneurisma tóraco-abdominal roto poupando a emergència das artérias abdominais. B: Controle angiográfico pós-operatório demonstrando a integridade do reparo e fluxo normal nas arterias abdominais. 
GONTIJO FILHO, B.; FANTINI, F. A.; BARBOSA, J. T.; PAULA E SILVA, J. A.; OLIVEIRA, C.; DRUMOND, L. F.; ALCOCER, E. P. VRANDECIC, M.O. - Cirurgia da aorta descendente e tóraco-abdominal com técnica de oclusăo proximal isolada da aorta ("open distal"). Rev. Bras. Cir. Cardiovasc., $10(3): 119-125,1995$.

descendente, a ressecçăo abordou o terço proximal ou médio da aorta em 17 ocasiōes; em 8 pacientes houve substituição da aorta torácica até o nivel do diafragma e em 1 portador de dissecção de aorta descendente foi necessário reimplante de artéria subclávia esquerda à prótese aórtica.

Nos casos de aneurismas tóraco-abdominais 4 pacientes não necessitaram reimplantes viscerais, pois o aneurisma poupava a origem do tronco celiaco (Figura 2). O tronco celiaco foi reimplantado seis vezes, sendo que em 2 pacientes estava ocluido (Figura 3), a artéria mesentérica superior cinco vezes e artéria renal direita e esquerda quatro vezes cada. Em 2 pacientes foi feito 0 implante de artéria intercostal próxima à região diafragmática, sendo o fluxo sangüineo restabelecido imediatamente após a anastomose. Nos pacientes com aneurisma da aorta descendente nāo foi realizado reimplante de artérias intercostais.

Um paciente portador de aneurisma tóracoabdominal roto apresentava doença aneurismática disseminada acometendo, também, a origem do tronco celíaco, da artéria mesentérica superior e da artéria renal esquerda. Nesse caso, a revascularizaçăo visceral necessitou a interposição de três segmentos de prótese reta $7 \mathrm{~mm}$, devido à grande distância entre a prótese aórtica e as artérias viscerais.

O tempo de oclusăo aórtica de toda série variou de 18 a 42 minutos, com média de 26 min. Nos pacientes portadores de aneurisma tóraco-abdominal realizamos a oclusão estadiada, liberando-se o fluxo arterial à medida em que cada anastomose era completada.

\section{RESULTADOS:}

Houve $4(11,4 \%)$ b́bitos no período hospitalar. Desses 4 óbitos, 3 (12\%) ocorreram no grupo de pacientes submetidos a substituiçāo da aorta descendente e apenas $1(10 \%)$ no grupo de aneurismas tóraco-abdominais. Considerando-se o quadro clínico pré-operatório, 3 dos 4 pacientes que faleceram apresentavam rotura aórtica, o que traduz uma mortalidade de $37,5 \%$ nos casos de rotura, contra uma mortalidade de $3,7 \%$ nos casos sem rotura. O outro óbito ocorreu em paciente com dor persistente e dissecçăo aguda de aorta descendente.

Com relação à causa determinante, houve 1 óbito per-operatório devido a fibrilação ventricular secundária a grave acidose metabólica. Tratava-se de paciente portador de aneurismas múltiplos, além de enorme aneurisma tóraco-abdominal roto, operado com quadro de insuficiência renal aguda. Dois pacientes evoluíram para falência de múltiplos ór-
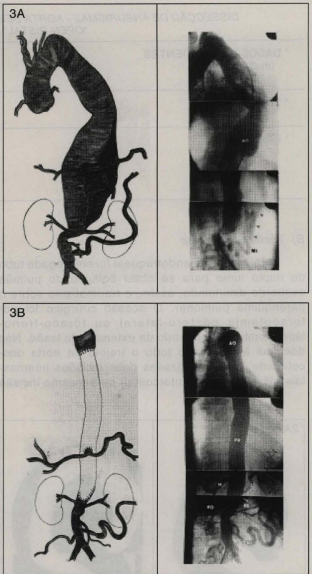

Fig. 3- A: Extenso aneurisma tóraco-abdominal com trombose parcial, levando à oclusăo do tronco celiaco. B: Aortografia pós. operatória demonstrando a extensa substituiçāo da aorta e fluxo normal pelo tronco celiaco que foi desobstruido $e$ reimplantado na prótese.

găos no pós-operatório, sendo que o primeiro apresentava insuficiência renal em diálise peritoneal $e$ o outro evoluiu com insuficiência respiratória e infecção pulmonar. O quarto óbito ocorreu em paciente com provável distúrbio acido-básico.

Nos demais pacientes encontramos, como complicações principais, os distúrbios respiratórios e neurológicos. Em 2 pacientes a assistência respiratória foi prolongada ( $>72$ horas), porém nāo houve necessidade de traqueostomia. Os distúrbios neurológicos foram graves e irreversíveis em $2(5,8 \%)$ pacientes que desenvolveram paraplegia e leve em outro que apresentou paresia transitória do membro inferior esquerdo. Ambos pacientes que apresenta- 
GONTIJO FILHO, B.; FANTINI, F. A.; BARBOSA, J. T.; PAULA E SILVA, J. A.; OLIVEIRA, C.; DRUMOND, L. F.; ALCOCER, E. P.; VRANDECIC, M. O. - Cirurgia da aorta descendente e tóraco-abdominal com técnica de oclusáo proximal isolada da aorta ("open distal"). Rev. Bras, Cir. Cardiovasc., 10 (3): 119-125, 1995.

ram paraplegia eram do grupo de aneurismas tóracoabdominais com tempo total de pinçamento superior a 40 minutos, e não haviam sido submetidos a reimplante de artérias intercostais. Uma paciente com paraplegia faleceu no $4^{2}$ més de pós-operatório, devido a tromboembolismo pulmonar.

\section{COMENTÁRIOS}

Um dos aspectos mais controvertidos da cirurgia cardiovascular refere-se ao melhor procedimento cirúrgico para tratamento das doenças da aorta torácica descendente e tóraco-abdominal.

Esta controvérsia deve-se à interferência de vários fatores, tais como: diversidade de doenças que acometem esse segmento aórtico, extensão do segmento aórtico acometido, presença de complicaçōes mórbidas importantes, tratamento na fase aguda do processo etc.

Dessa forma os resultados cirúrgicos relatados apresentam variaçōes importantes com taxas de mortalidade que oscilam de $3 \%$ a $21 \% 1,11$ ou, mesmo, das graves complicaçōes neurológicas com índices de $0 \%$ a $28 \%$, chegando até $40 \%$ em casos de dissecçāo aórtica ${ }^{8}$.

Várias técnicas têm sido propostas para a substituição do segmento aórtico acometido, desde a forma clássica de ressecção aórtica com substituição por prótese e sutura direta, às substituições por próteses intraluminais, aos desvios extra-anatômicos com trombo-exclusão da aorta, às fenestrações $\theta$. mais recentemente, às substituições endovasculares por cateterismo. As técnicas alternativas, como a trombo-exclusão e as fenestraçōes nos casos de dissecção aórtica, têm sido utilizadas por poucos grupos cirúrgicos. Nossa experiência com a tromboexclusão ${ }^{7}$, apesar de apresentar resultados iniciais satisfatórios, não foi a mesma a longo prazo. Houve casos de evoluçăo da doença no segmento aórtico abdominal, cuja correção foi extremamente dificultada pelo próprio método; além disso, 2 pacientes apresentavam pseudo-aneurisma com infecçāo e rotura do coto aórtico em fundo cego. Já as fenestraçōes distais, que podem ser úteis em casos de dissecçăo aórtica aguda com isquemia visceral ou de membros inferiores, aplicam-se a um número muito limitado de pacientes portadores de doenças da aorta torácica. Além disso, a dissecçăo aórtica permanece em toda sua extensão com todos os outros riscos. De um modo geral, pode-se dizer, portanto, que a grande maioria dos relatos referese à substituiçăo clássica com sutura direta, havendo maior divergência quanto à necessidade ou não de se promover apoio circulatório à parte distal da aorta durante o período de sua oclusāo.
Esse apoio circulatório pode ser obtido através de circulaçāo extracorpórea (by-pass átrio esquerdo - femoral, ou by-pass fêmoro-femoral), ou por um shunt temporário passivo como do tipo Gott. Os autores que sustentam a perfusão aórtica distal relatam menor índice de complicaçōes renais, meIhor controle da pressão arterial com menor sobrecarga imposta ao ventrículo esquerdo, menor índice de hipotensăo arterial a acidose metabólica durante a liberação da pinça aórtica e menos complicaçōes neurológicas ${ }^{2}$.

NAJAFI 10 relata sua mudança de estratégia, abandonando a técnica de oclusão aórtica isolada, que usou até 1980, qando teve 1 caso de paraplegia após um periodo relativamente curto de isquemia medular (28 minutos). Passou a usar 0 by-pass fêmoro-femoral, que se tornou sua técnica de escolha. Relata que os possiveis problemas associados ao sistema de circulaçăo extracorpórea completo, inclusive oxigenadores, năo ocorreram em sua série de 37 pacientes, devido ao alto padrăo tecnológico alcançado por estes materiais. SEJESSER ot alii ${ }^{13}$, corroborando este fato, relataram melhores resultados em um grupo de 49 pacientes operados com sistemas de tubo heparinizados e baixa dose sistêmica de heparina do que o grupo operado apenas com oclusão simples $(n=42)$, inclusive com menor indice de sangramento pós-operatório. VERDANT et alii 14 empregando o shunt de Gott em 173 pacientes, relataram mortalidade de $15 \%$ e, surpreendentemente, ausência de complicaçōes neurológicas. Há que se considerar, entretanto, que a grande maioria dos seus pacientes foi submetida a ressecçāo limitada ao terço proximal e médio da aorta descendente, o que, por si só, diminuiu o risco de complicaçöes neurológicas, conforme comentou Crawford na mesma publicação. BORST et alii ${ }^{1}$, utilizando a técnica de by-pass átrio esquerdo-femoral em 132 pacientes, reiatam um excelente resultado com mortalidade de $3 \%$ e de dano neurológico irreversivel em apenas $2,3 \%$ dos casos, numa série que incluiu ressecçōes extensas da aorta torácica.

Contrapondo-se a estes achados, alguns grupos cirúrgicos não verificaram grandes diferenças entre os pacientes operados com apoio circulatório e aqueles operados com oclusão isolada da aorta. LIVESAY et alii ${ }^{9}$ apresentaram melhores resultados em 263 pacientes, operados com oclusão simples da aorta do que em 97 pacientes operados com suporte circulatório, sendo que neste último grupo ainda predominaram os problemas hemorrágicos.

CRAWFORD et ali 5 , em 543 pacientes submetidos a substituição da aorta torácica, relatam $6 \%$ de complicaçōes neurológicas no grupo operado com circulaçăo extracorpórea (153 pacientes) e $5 \%$ no 
GONTIJO FILHO, B.; FANTINI, F. A.; BARBOSA, J. T.; PAULA E SILVA, J. A.; OLIVEIRA, C.; DRUMOND, L. F.; ALCOCER, E. P.; VRANDECIC, M. O. - Cirurgia da aorta descendente e tóraco-abdominal com técnica de oclusăo proximal isolada da aorta ("open distal"). Rev. Bras, Cir. Cardiovasc., 10 (3): 119-125, 1995.

grupo operado com oclusăo isolada (390 pacientes) ${ }^{\star}$. Em outro relato, confirmam os mesmos achados, além de não observarem melhora dos resultados com a monitorizaçāo de potenciais somato. sensoriais.

SCHEPENS et alii ${ }^{12}$, em uma série de 88 pacientes submetidos a correçăo de aneurisma tóraco-abdominal, năo encontraram diferenças quanto aos problemas neurológicos com relaçăo à técnica cirúrgica empregada, embora no grupo com circulaçăo extracorpórea com biopump o indice de outras complicaçōes (insuficiència renal, acidose) tenha sido menor. COOLEY ${ }^{3}$, em 1990 , propôs uma técnica alternativa que chamou de "open distal", na qual, após posicionamento da pinça aórtica proximal, a aorta era incisada sem controle da sua parte inferior, sendo todo o sangue recolhido a um reservatório $e$, entāo, reinfundido por uma cânula colocada na veia femoral.

Desta forma, evita-se a sobrecarga aguda imposta ao ventrículo esquerdo e, ao se promover a exsangüinaçăo do paciente, passa-se a controlar a pressăo arterial pela sua volemia. Além disso, consegue-se o reaproveitamento imediato do sangue aspirado do campo cirúrgico. Em um relato posterior COOLEY \& BALDWIN ${ }^{4}$ apresentam os resultados obtidos em um grupo de 55 pacientes operados com oclusāo simples $(n=31)$ ou com a nova técnica $(n=24)$. Embora a mortalidade fosse semeIhante nos dois grupos $(13 \%$ e $17 \%$, respectivamente), houve menos complicaçōes neurológicas no segundo grupo, possivelmente devido a diminuição da pressão do líqüido cérebro-espinhal observada nesses pacientes. Os nossos resultados em 35 operaçōes com a técnica "open-distal" foram semeIhantes, com uma mortalidade hospitalar de $11,4 \%$ e com $2(5,8 \%)$ casos de paraplegia. Aprofundando esta análise do nosso grupo, a condiçāo clínica préoperatória e a extensão da doença aórtica com conseqũente aumento do tempo de isquemia medular foram fatores determinantes de maior morbimortalidade.

Os 2 casos de paraplegia ocorreram em aneurismas tóraco-abdominais extensos com período de oclusão aórtica acima de 40 minutos. Por outro lado, a mortalidade hospitalar foi muito maior nos pacientes que apresentavam rotura aórtica com complicaçōes pré-operatórias. Com relaçāo à função renal, podemos concluir que a técnica foi eficiente. Apenas os pacientes que apresentavam insuficiência renal estabelecida no pré-operatório evoluíram mal, tendo 1 falecido na sala de operação e outro no pós-operatório imediato com falência de múltiplos órgāos.

A funçăo renal dos demais pacientes foi preservada sem necessidade de diálise.

Promovendo-se uma análise dos diversos relatos da literatura referentes ao tratamento cirúrgico das doenças da aorta torácica e tóraco-abdominal, encontramos alguns pontos que coincidem com a nossa experiência.

1) A técnica da ressecçăo com substituiçăo do segmento aórtico ainda é a mais usada, uma vez que outras como fenestração e tromboexclusāo nāo eliminaram o risco de complicaçōes e associam-se a problemas inerentes à própria técnica, principalmente na evoluçāo a médio e longo prazos.

2) A condiçăo clínica pré-operatória, a extensāo da doença aórtica e o tempo de isquemia sāo fatores determinantes de maior morbimortalidade em todas as técnicas utilizadas.

3) A técnica "open-distal" apresenta resultados melhores que os obtidos com a oclusão simples da aorta. Com relação às técnicas que empregam apoio circulatório distal, a técnica "open-distal" apresentou resultados semelhantes, considerando-se os casos de ressecçăo de aorta descendente e limitados a um período de isquemia inferior a $30 \mathrm{mi}-$ nutos. Nos casos mais extensos e com períodos isquêmicos maiores, as técnicas com apoio circulatório parecem apresentar alguma vantagem.

4) Novos métodos como a hipotermia profunda sistêmica ou regional ${ }^{8}$ devem ser investigados com maior profundidade, para redução efetiva dos índices de lesōes neurológicas decorrentes da isquemia medular.

- Discussăo em Ann. Thorac. Surg., 46: 147-154, 1988. 
GONTIJO FILHO, B.; FANTINI, F. A.;BARBOSA, J. T.; PAULA E SILVA, J. A.; OLIVEIRA, C.; DRUMOND, L. F.; ALCOCER, E. P.; VRANDECIC, M. O. - Cirurgia da aorta descendente e tóraco-abdominal com técnica de oclusâo proximal isolada da aorta ("open distal"). Rev. Bras. Cir. Cardiovasc., 10 (3): 119-125, 1995.

RBCCV $44205-267$

GONTIJO FILHO, B.; FANTINI, F. A.; BARBOSA, J. T.; PAULA E SILVA, J. A.; OLIVEIRA, C.; DRUMOND, L.F.; ALCOCER, E.P.; VRANDECIC, M. O. - Surgical treatment of descending thoracic and thoracoabdominal aorta with the "open distal" technique. Rev. Bras. Cir. Cardiovasc., 10 (3): 119-125, 1995.

ABSTRACT: Since Jan/91 we have been using the "open distal" technique (ODT) described by Cooley, for surgical treatment of diseases of the descending thoracic and thoracoabdominal aorta. From jan/91 to jan/ 95 , the technique was used in 35 instances ( 25 for descending aorta and 10 for thoracoabdominal aorta). Degenerative aneurysms and aortic dissections had similar incidence in this group ( $48.5 \%$ and $40.0 \%$, respectively). In 8 patients the aorta was ruptured which was partially occluded by the lung (5 cases). abdominal organs (2 cases) and esophagus (1 case). Surgical exposure was obtained either by left thoracotomy or thoraco-phreno-laparotomy. Heparin was used in a dosis of $1.5 \mathrm{mg} / \mathrm{kg}$ and all the blood was collected from the patient and returned through the femoral vein. There were $4(11.4 \%)$ hospital deaths and 2 $(5,8 \%)$ patients with thoracoabdominal aneurysms developed paraplegia. Morbi - mortality of the group was related to the pre-operative clinical condition and extension of the disease. In our opinion ODT is a simple and effective surgical technique for diseases of the descending thoracic and thoraco-abdominal aorta. Maybe for extensive resections with long aortic cross clamping time, other methods providing a better spinal cord protection should be evaluated.

DESCRIPTORS: Aorta, descending, surgery. Aorta, thoracoabdominal, surgery. Cooley technique, "open distal", "Open distal", Cooley technique.

\section{REFERÊNCIAS BIBLIOGRÁFICAS}

1 BORST, H. G.; JURMANN, M.; BUHNER, B.; LAAS, J. - Risk of replacement of descending aorta with a standardized left heart by-pass technique. J. Thorac. Cardiovasc. Surg., 107: 126-133, 1994

2 CARTIER, R.; ORSZULAK, T. A.; PAIROLERO, P. C.; SCHAFF, H. V. - Circulatory support during cross clamping of the descending thoracic aorta: evidence of improved organ perfusion. J. Thorac. Cardiovasc. Surg., 99: 1038-1047, 1990.

3 COOLEY, D. A. - Surgical management of aortic dissection. Texas Heart Inst. J., 4: 289-301, 1990.

4 COOLEY, D. A. \& BALDWIN, R. T. - Technique of open distal anastomosis for repair of descending thoracic aortic aneurysms. Ann. Thorac. Surg., 54: 932-936, 1992.

5 CRAWFORD, E. S.; MIZRAHI, E. M.; HESS, K. R.; COSELLI, J. S.; SAFI, H. H.; PATEL, V. M. - The impact of distal aortic perfusion and somato-sensory evoked potential monitoring on prevention of paraplegia after aortic aneurysm operation. $J$. Thorac. Cardiovasc. Surg., 95: 357-367, 1988.

6 ELEFTERIADES, J. A.; HARTLEROAD, J.; GUSBERG, R. J.; SALAZAR, A. M.; BLACK, H. R.; KOPF, G. S.; BALDWIN, J. C.; HAMMOND, G. L. - Long-term experience with descending aortic dissection: the complication specific approach. Ann. Thorac. Surg., 53: 11-21, 1992.

7 GONTIJO FILHO, B.; BARBOSA, J. T.; PAULA E SIL. VA, J. A.: FANTINI, F. A.; SALUN, M. A.: VRANDECIC, M. O. - Correçăo cirúrgica de aneurisma da aorta torácica por têcnica de exclusăo. Rev. Bras. Cir. Cardiovasc., 3: 65-72, 1988.
8 KOUCHOUKOS, N. T.; WAREING, T. H.; IZUMUTO, H.; KLAUSING, W.; ABBOUD, N. - Elective hypothermic cardiopulmonary bypass and circulatory arrest for spinal cord protection during operations on the thoracoabdominal aorta. J. Thorac. Cardiovasc. Surg., 99: 659-664, 1990.

9 LIVESAY, J. J.; COOLEY, D. A.; VENTEMIGLIA, R. A.; MONTERO, C. G.; WARRIAN, R. K.; BROWN, D. M.; DUNCAN, J. M. - Surgical experience in descending thoracic aneurysmectomy with and without adjucts to avoid ischemia. Ann. Thorac. Surg., 39: 37-46, 1985.

10 NAJAFI, H. - 1993 Update. Ann. Thorac. Surg., 56: 1042-1045, 1993.

11 REUL, G. J.; COOLEY, D. A.; HALLMAN, G. L.; REDDY, S. B.; KYGER, E. R.; WUKASCH, D. C. - Dissecting aneurysm of the descending aorta. Arch. Surg., 110: $632-640,1975$.

SCHEPENS, M. A. A. M.; DEFAUW, J. J. A. M.; HAMERLIJNCK, R. P. H. M.; DE GEEST, R.; VERMEULEN, F. E. E. - Surgical treatment of thoracoabdominal aortic aneurysms by simple cross clamping; risk factors and late results $J$. Thorac. Cardiovasc. Surg., 107: 134-142, 1994.

13 SEGESSER, L. K.; KILLER, I.; JENNI, R.; LUTZ, U.; TURINA, M. I. - Improved distal circulatory support for repair of descending thoracic aortic aneurysms. Ann. Thorac. Surg., 56: 1373-1380, 1993.

14 VERDANT, A.; PAJÉ, A.; COSETTE, R.; DONTIGNY, L; PAJE, P; BAILLOT, R. - Surgery of the descending thoracic aorta: spinal cord protection with the Gott shunt. Ann. Thorac. Surg., 46: 147-154, 1988. 